Case Report

\title{
Improvement of Renal Function by Long-Term Sustained Eculizumab Treatment in a Patient with Paroxysmal Nocturnal Hemoglobinuria
}

\author{
Haruhiko Ninomiya, ${ }^{1}$ Naoshi Obara, ${ }^{2}$ Akiko Niiori-Onishi, ${ }^{3}$ Yasuhisa Yokoyama, ${ }^{2}$ \\ Mamiko Sakata-Yanagimoto, ${ }^{2}$ Yuichi Hasegawa, ${ }^{2}$ and Shigeru Chiba ${ }^{2}$ \\ ${ }^{1}$ Department of Medical Sciences, Faculty of Medicine, University of Tsukuba, Tsukuba 305-8575, Japan \\ ${ }^{2}$ Department of Hematology, Faculty of Medicine, University of Tsukuba, Tsukuba 305-8575, Japan \\ ${ }^{3}$ Department of Internal Medicine, Hitachi, Ltd., Hitachinaka General Hospital, Hitachinaka 312-0057, Japan \\ Correspondence should be addressed to Haruhiko Ninomiya; ninomiya.haruhiko.ft@u.tsukuba.ac.jp
}

Received 4 April 2015; Accepted 27 May 2015

Academic Editor: Ramon Tiu

Copyright ( 2015 Haruhiko Ninomiya et al. This is an open access article distributed under the Creative Commons Attribution License, which permits unrestricted use, distribution, and reproduction in any medium, provided the original work is properly cited.

Chronic kidney disease (CKD) is one of the major manifestations of paroxysmal nocturnal hemoglobinuria (PNH). CKD in PNH is induced mainly by intravascular hemolysis of PNH-affected red blood cells (RBC) missing the glycosylphosphatidylinositolanchored proteins with complement-regulatory activities, CD55 and CD59. CKD develops by heme absorption in the proximal tubules resulting in the interstitial deposition of iron in the kidneys. We administered eculizumab to a patient with $\mathrm{PNH}$, who was one of 29 patients enrolled in the AEGIS clinical trial, an open-label study of eculizumab in Japan. The patient was complicated by stage 3 CKD with impaired estimated glomerular filtration rate (eGFR), at grade G3b, and had obvious proteinuria (2-3+, 1-2 g/day). In a two-year extension to the 12-week AEGIS study, eGFR improved significantly, and the eGFR has since been maintained at grade G2 without proteinuria by sustained eculizumab treatment ( $>6$ years). Renal function improved and maintained by longterm sustained eculizumab treatment, presumably by clearance of iron from the kidney as well as inhibition of the production of anaphylatoxin C5a, even in advanced stages of CKD, is one of the benefits of eculizumab treatment in PNH.

\section{Introduction}

Renal failure is one of the important factors which determine the prognosis of paroxysmal nocturnal hemoglobinuria $(\mathrm{PNH})$, especially for Japanese patients. It has been reported that renal failure is responsible for $18 \%$ of deaths and is a significant risk factor for Japanese PNH patients [1]. Renal dysfunction in $\mathrm{PNH}$ is believed to be related to microvascular thrombosis in the glomeruli and hemoglobinuria, resulting in the interstitial deposition of iron in the kidneys [2].

The first clinical investigation of eculizumab was an openlabel, 12-week, Phase II pilot study that enrolled 11 patients in two UK study centers [3]. Hillmen et al. reported that $64 \%$ of PNH patients showed evidence of chronic kidney disease (CKD) [4]. They reported that long-term sustained eculizumab treatment resulted in a time-dependent improvement of renal function in PNH; 93.1\% of patients exhibited improvement (44.8\%) or stabilization (48.3\%) in CKD score at 36 months [5]. Improvement in renal function was more commonly seen in patients with baseline stages 1-2 CKD, although improvement was also observed in patients with stages 3-5 CKD [4]. Long-term observation of Japanese PNH patients in a two-year extension to the 12-week AEGIS study also showed that the majority of patients exhibited stable (56\%) or improved (41\%) renal function [6].

Here, we report a long-term ( $>6$ years) observation of a $\mathrm{PNH}$ patient, with baseline stage $3 \mathrm{CKD}$, who was enrolled in the AEGIS clinical trial [7], and demonstrated the maintenance effect of eculizumab on renal function. 


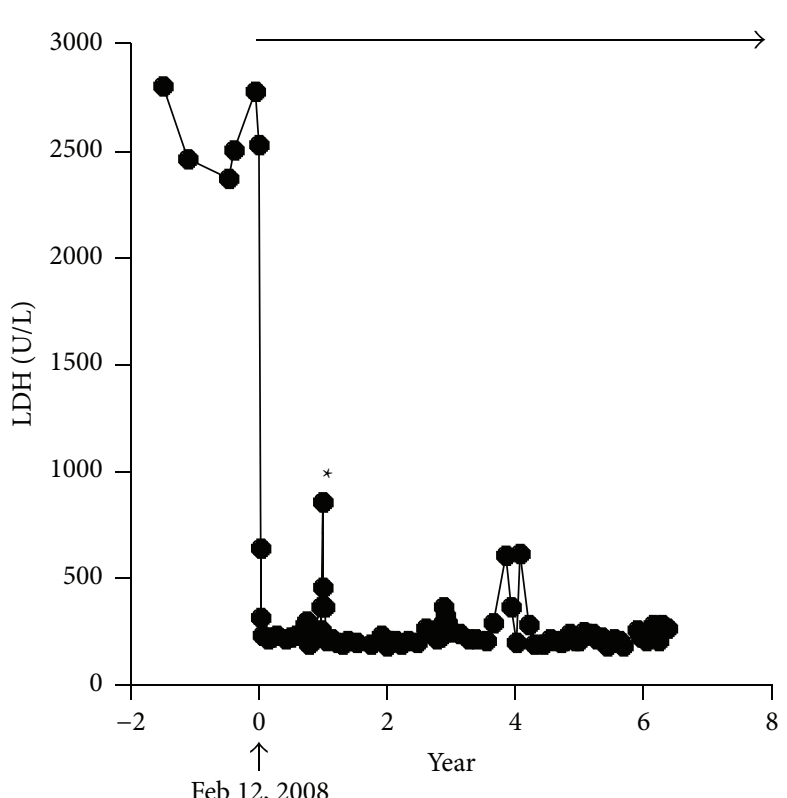

(a)

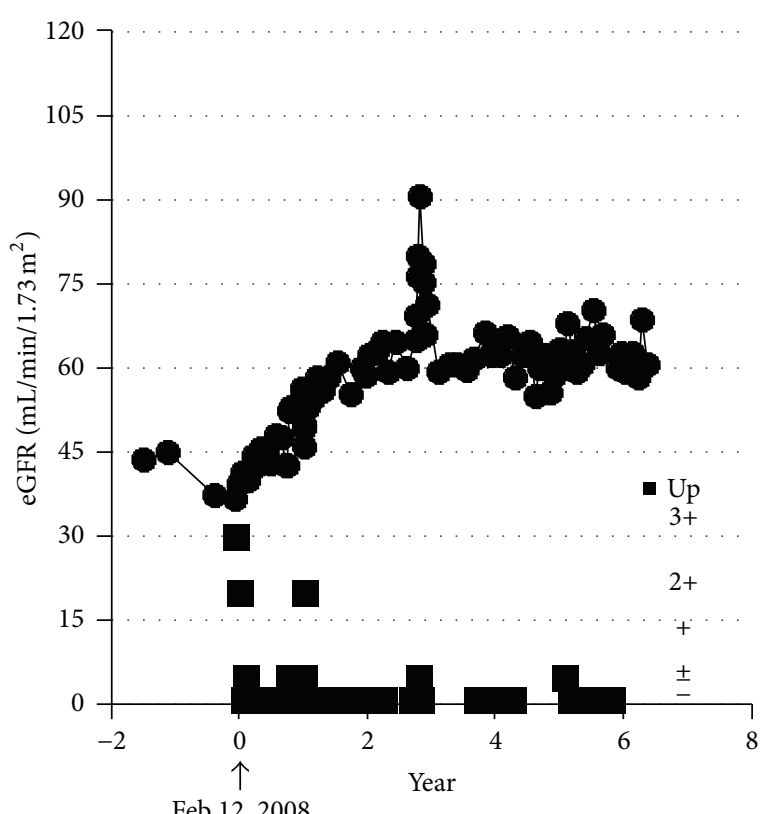

(b)

FIGURE 1: Clinical course of the patient. On February 12, 2008, he was enrolled in the AEGIS clinical trial. (a) Administration of eculizumab resulted in a rapid reduction of LDH. Asterisk indicates the increased LDH due to delayed administration of eculizumab during an AE of hyperbilirubinemia. Arrow indicates eculizumab treatment. (b) He was complicated with stage 3 CKD, grade G3b, and obvious proteinuria, at baseline. Eculizumab treatment improved eGFR gradually. Since two years after introduction of eculizumab, eGFR at grade G2 and no proteinuria have been maintained. Closed squares denote grades of proteinuria $(-, \pm,+, 2+$, and $3+)$.

\section{Case Report}

A 70-year-old male PNH patient was enrolled in an openlabel study of eculizumab, as one of 29 patients enrolled in the AEGIS clinical trial in Japan [7]. The trial was sponsored by Alexion Pharmaceuticals. His disease was diagnosed on admission to a regional hospital due to urinary tract infection, two years before enrollment in the study. At the time of diagnosis, laboratory data indicated pancytopenia, leukocytes $3 \times 10^{9} / \mathrm{L}$, hemoglobin $(\mathrm{Hb}) 7.7 \mathrm{~g} / \mathrm{dL}$, platelet $99 \times 10^{9} / \mathrm{L}$, total bilirubin $2.7 \mathrm{mg} / \mathrm{dL}$, and $886 \mathrm{U} / \mathrm{L} \mathrm{LDH}$. A chromosomal analysis of bone marrow cells revealed an abnormality, $46 \mathrm{XY}, \mathrm{t}(1 ; 7)$ (q44;q11), in 4 of 20 cells. A flow cytometric study of peripheral blood revealed significant ratios of PNH-affected, CD59-deficient, red blood cells (RBC) (29.5\%), neutrophils (86.5\%), and monocytes (88.9\%). A diagnosis of $\mathrm{PNH}$ with myelodysplastic syndrome was made. Before enrollment in the AEGIS clinical trial, constant $\mathrm{RBC}$ transfusions (2-4 U/month) had been required. No thromboembolic episodes had been demonstrated. Before the diagnosis of $\mathrm{PNH}$, he had an almost ten-year past history of diabetes mellitus (DM), which had been well controlled by insulin therapy (glycoalbumin 18-21\%).

Administration of eculizumab (initiated on February 12, 2008) resulted in rapid reduction of $\mathrm{LDH}$ levels, and he became RBC transfusion-independent, indicating efficient inhibition of intravascular hemolysis by eculizumab. He was hospitalized twice (January to February, 2009, and November to December, 2010) due to hyperbilirubinemia, due to uncertain causes. These serious adverse events (AE) were assessed as unrelated to the eculizumab treatment, because continuation of eculizumab administration did not worsen the AE. No obstructive biliary tract lesions or known hepatic virus infections were detected by laboratory examinations, and he recovered from the AE spontaneously on both occasions.

As shown in Figure 1, at the time of enrollment in the AEGIS clinical trial, an impaired estimated glomerular filtration rate (eGFR) with obvious proteinuria (1-2 g/day), grade G3b (eGFR $30-45 \mathrm{~mL} / \mathrm{min} / 1.73 \mathrm{~m}^{2}$ ) according to the modified 2012 KDIGO CKD guideline adopted for the Japanese [8], was seen. In a two-year extension to the 12-week AEGIS study, renal function gradually improved to stage 2 CKD, grade G2 (eGFR $60-90 \mathrm{~mL} / \mathrm{min} / 1.73 \mathrm{~m}^{2}$ ), without proteinuria, which was maintained by sustained eculizumab treatment. Proteinuria disappeared soon after the initiation of eculizumab (Figure 2). Improved eGFR levels and no proteinuria have been maintained throughout the 6-year eculizumab treatment (until the patient was 77 years old). Proximal tubular dysfunction was not apparent by the urinary level of liver-type fatty acid binding protein $(2.5 \mu \mathrm{g} / \mathrm{g} \cdot \mathrm{Cr})$. Serum ferritin levels were increased both before $(459 \mathrm{ng} / \mathrm{mL})$ and after six years of treatment with eculizumab $(547 \mathrm{ng} / \mathrm{mL}$, June 2014). Because the patient's DM had been well controlled by insulin therapy, this improvement in renal function seems to have been induced by the long-term administration of eculizumab, which inhibited intravascular hemolysis and hemoglobinuria (renal hemosiderosis). 


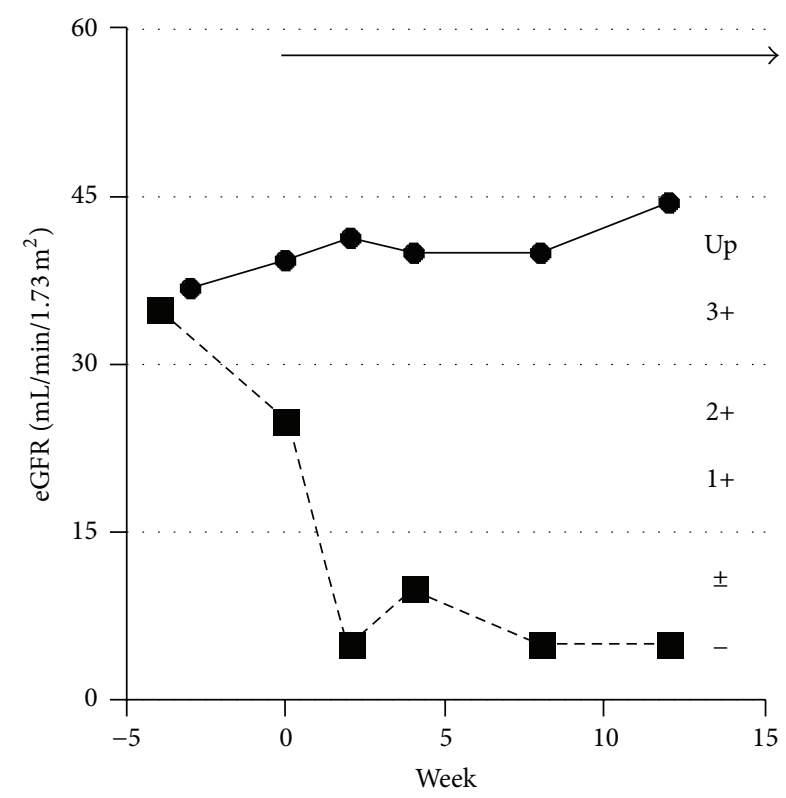

FIgURE 2: eGFR and proteinuria around the initiation of eculizumab. Obvious proteinuria (closed squares, up), and quickly disappearing proteinuria by initiation of eculizumab treatment (arrow). Closed circles denote eGFR.

\section{Discussion}

Chronic intravascular hemolysis results in iron deposition in the kidney in almost all PNH patients. This excess iron can be detected by magnetic resonance imaging $[9,10]$. Excess iron deposition can result in proximal tubular dysfunction $[2,11,12]$. Chronic renal failure due to hemosiderosis and subsequent interstitial scarring may occur in patients with long-standing PNH $[2,13]$. Inhibition of production of anaphylatoxin C5a by eculizumab is another possible mechanism improving the glomerular function of $\mathrm{PNH}$, as reported in cases of dense deposit disease and C3 glomerulonephritis [14].

Improvement of renal function in $\mathrm{PNH}$ by administration of eculizumab is time-dependent, as reported by Hillmen et al. [5] and Kanakura et al. [6]. Improvement of renal function is less common in the advanced stages $3-5$ of CKD than stages 1-2 of CKD, even with long-term administration of eculizumab, whereas patients with stages 3-5 did not worsen during treatment with eculizumab [4]. Improvement of renal function in our case may be partly explained due to the initiation of eculizumab treatment relatively soon after the onset of PNH. At the time of enrollment in the AEGIS clinical trial, the ratio of $\mathrm{PNH}$-affected $\mathrm{RBC}$ was $<30 \%$, which suggests a relatively small amount of iron may have been deposited in the kidneys. The antithrombotic effects and anticomplement activity (inhibition of C5a production) of eculizumab also contributed to the improvement of glomerular function, because obvious proteinuria disappeared soon after administration (Figure 2).

Our case shows the clinical effects of eculizumab on the improvement of renal function even in patients with advanced stage CKD. When eculizumab treatment is introduced at an early stage of $\mathrm{PNH}$, prognosis may be improved by recovery of renal function.

\section{Conflict of Interests}

Haruhiko Ninomiya has received honoraria for lectures from Alexion Pharmaceuticals, and Shigeru Chiba works for an institution that has received research funding from Alexion Pharmaceuticals.

\section{References}

[1] J.-I. Nishimura, Y. Kanakura, R. E. Ware et al., "Clinical course and flow cytometric analysis of paroxysmal nocturnal hemoglobinuria in the United States and Japan," Medicine, vol. 83, no. 3, pp. 193-207, 2004.

[2] D. A. Clark, S. A. Butler, V. Braren, R. C. Hartmann, and D. E. Jenkins Jr., "The kidneys in paroxysmal nocturnal hemoglobinuria," Blood, vol. 57, no. 1, pp. 83-89, 1981.

[3] P. Hillmen, C. Hall, J. C. W. Marsh et al., "Effect of eculizumab on hemolysis and transfusion requirements in patients with paroxysmal nocturnal hemoglobinuria," The New England Journal of Medicine, vol. 350, no. 6, pp. 552-559, 2004.

[4] P. Hillmen, M. Elebute, R. Kelly et al., "Long-term effect of the complement inhibitor eculizumab on kidney function in patients with paroxysmal nocturnal hemoglobinuria," American Journal of Hematology, vol. 85, no. 8, pp. 553-559, 2010.

[5] P. Hillmen, P. Muus, A. Röth et al., "Long-term safety and efficacy of sustained eculizumab treatment in patients with paroxysmal nocturnal haemoglobinuria," British Journal of Haematology, vol. 162, no. 1, pp. 62-73, 2013.

[6] Y. Kanakura, K. Ohyashiki, T. Shichishima et al., "Long-term efficacy and safety of eculizumab in Japanese patients with PNH: AEGIS trial," International Journal of Hematology, vol. 98, no. 4, pp. 406-416, 2013.

[7] Y. Kanakura, K. Ohyashiki, T. Shichishima et al., "Safety and efficacy of the terminal complement inhibitor eculizumab in Japanese patients with paroxysmal nocturnal hemoglobinuria: the AEGIS Clinical Trial," International Journal of Hematology, vol. 93, no. 1, pp. 36-46, 2011.

[8] S. Matsuo, E. Imai, M. Horio et al., "Revised equations for estimating glomerular filtration rate (GFR) from serum creatinine in Japan," American Journal of Kidney Diseases, vol. 53, no. 6, pp. 982-992, 2009.

[9] K. Suzukawa, H. Ninomiya, S. Mitsuhashi, I. Anno, T. Nagasawa, and T. Abe, "Demonstration of the deposition of hemosiderin in the kidneys of patients with paroxysmal nocturnal hemoglobinuria by magnetic resonance imaging," Internal Medicine, vol. 32, no. 9, pp. 686-690, 1993.

[10] D. Mathieu, A. Rahmouni, P. Villeneuve, M. C. Anglade, H. Rochant, and N. Vasile, "Impact of magnetic resonance imaging on the diagnosis of abdominal complications of paroxysmal nocturnal hemoglobinuria," Blood, vol. 85, no. 11, pp. 3283-3288, 1995.

[11] A. L. Riley, L. M. Ryan, and D. A. Roth, "Renal proximal tubular dysfunction and paroxysmal nocturnal hemoglobinuria," The American Journal of Medicine, vol. 62, no. 1, pp. 125-129, 1977. 
[12] P.-J. Hsiao, S.-C. Wang, M.-C. Wen, L.-K. Diang, and S.-H. Lin, "Fanconi syndrome and CKD in a patient with paroxysmal nocturnal hemoglobinuria and hemosiderosis," American Journal of Kidney Diseases, vol. 55, no. 1, pp. el-e5, 2010.

[13] P. Zachee, M. Henckens, B. van Damme, M. A. Boogaerts, H. Rigauts, and R. K. A. Verberckmoes, "Chronic renal failure due to renal hemosiderosis in a patient with paroxysmal nocturnal hemoglobinuria," Clinical Nephrology, vol. 39, no. 1, pp. 28-31, 1993.

[14] L. C. Herlitz, A. S. Bomback, G. S. Markowitz et al., "Pathology after eculizumab in dense deposit disease and C3 GN," Journal of the American Society of Nephrology, vol. 23, no. 7, pp. 12291237, 2012. 


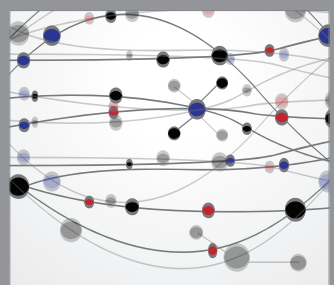

The Scientific World Journal
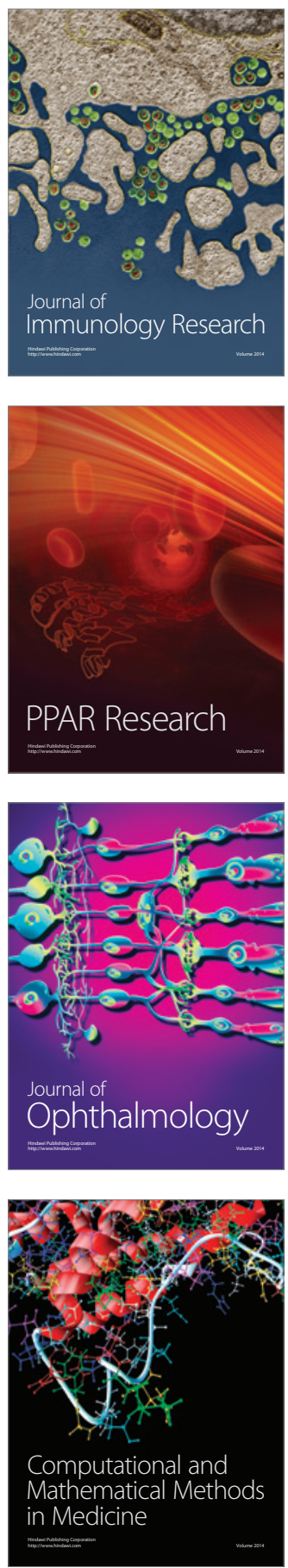

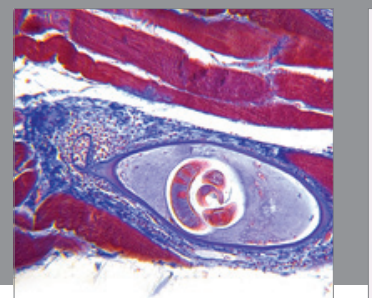

Gastroenterology

Research and Practice
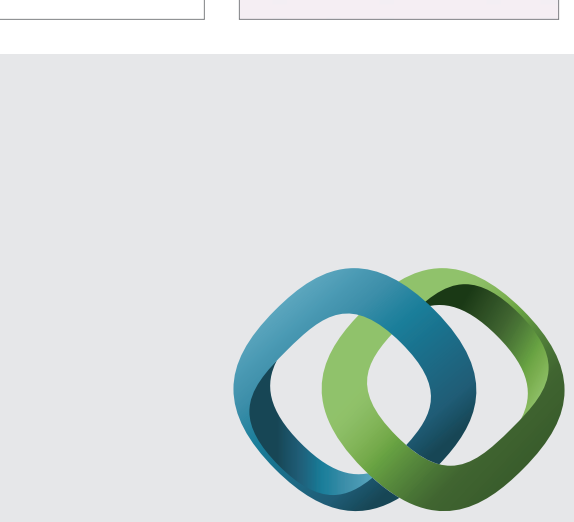

\section{Hindawi}

Submit your manuscripts at

http://www.hindawi.com
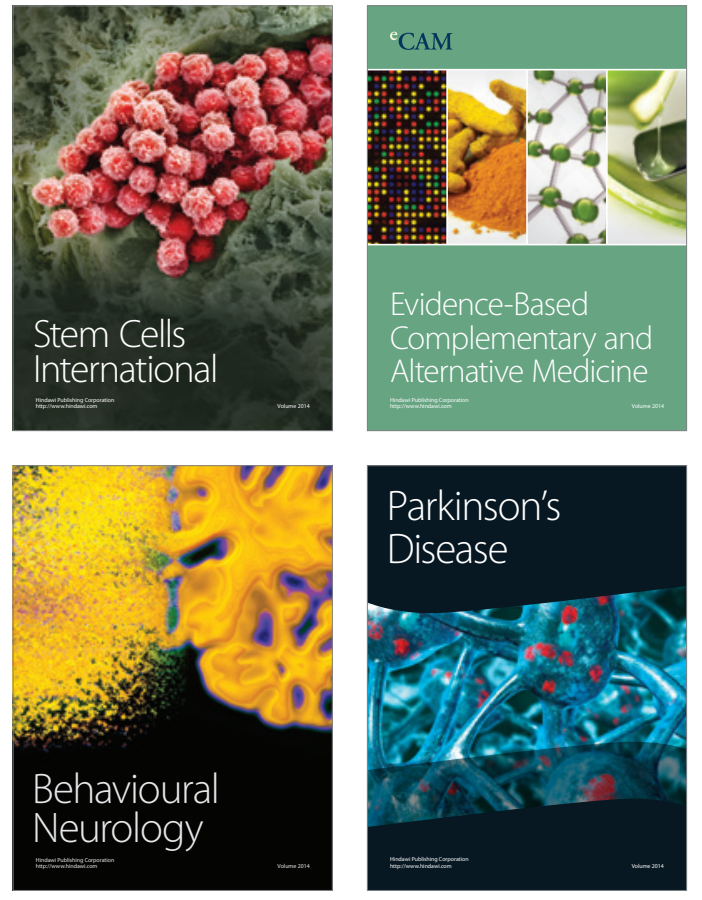
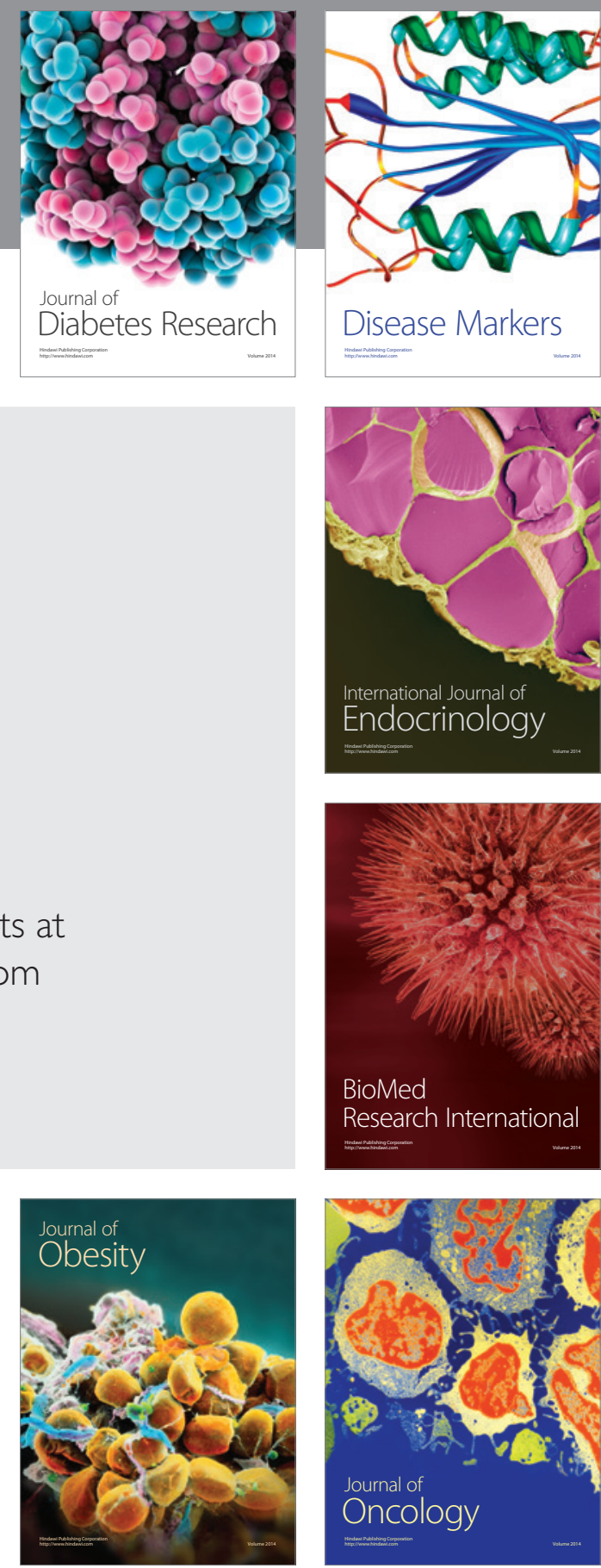

Disease Markers
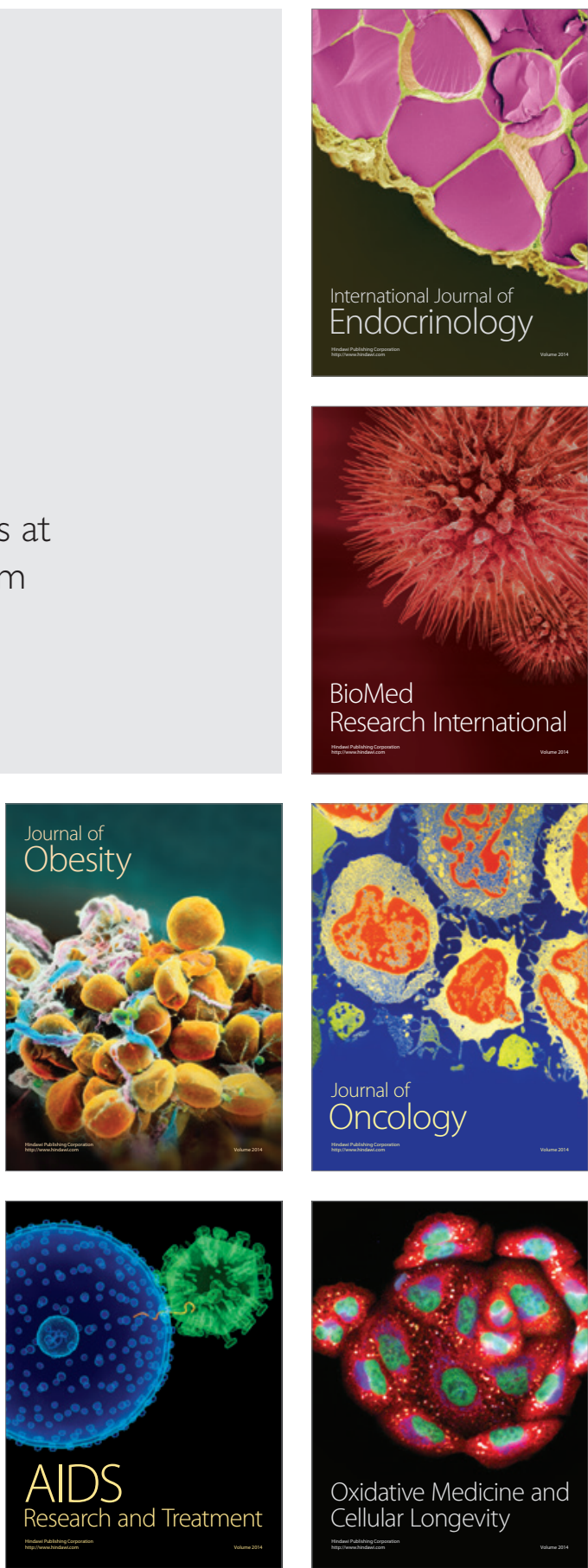